\title{
The Neurocognitive Basis of Compromised Autonomy After Traumatic Brain Injury: Clinical and Ethical Considerations
}

\author{
Warren E. Lux \\ Program in Human Research Ethics, Office of the Science Advisor, U.S. Environmental Protection Agency, Washington, DC, \\ and Center for Clinical Bioethics, Georgetown University, Washington, DC
}

\begin{abstract}
Summary: It is widely accepted, based on the principle of respect for autonomy, that there are ethical constraints on the range of tactics that persons may use to influence the decisions and behaviors of others. However, accurate ascriptions of autonomy to either persons or acts may vary considerably, depending on properties of the person, the situation, or both. Traumatic brain injury affects cognitive domains that are critical for the effective exercise of autonomy, and so offers a context for further examination of this variability. Analysis of
\end{abstract}

the neuropathology of traumatic brain injury and its neurocognitive consequences provides a foundation for understanding cases in which autonomy is compromised even though legal competency may be preserved. Respecting autonomy in these cases is not always straightforward; it may entail both special ethical obligations and consideration of tactics that would not be morally permissible under other circumstances. Key Words: Autonomy, axonal strain injury, executive function, self-assessment, self-awareness, traumatic brain injury.

\section{INTRODUCTION}

Autonomy is a word derived from the Greek for 'self' (autos) and 'law' (nomos), and central to the concept is the idea of self-governance. ${ }^{1,2}$ Both acts, including choices, and persons may be referred to as autonomous; in both instances, genuine self-guidance free from external control is essential for full autonomy. Respect for autonomy, particularly as it relates to treatment choices, is one of the core principles of biomedical ethics. ${ }^{2}$

Implicit in the attitudes that underlie the way in which this principle is applied in many, if not most, chronic stable clinical circumstances encountered in American healthcare is the assumption that legally competent adults in our society can properly be regarded as substantially autonomous persons. As a consequence, such adults are generally allowed to make treatment decisions on their own behalf, and there are ethical, as well as legal, constraints on what others are permitted to do to influence those decisions or to impede or alter the behaviors that flow from them. Physical restraint, administration of drugs to modify behavior without consent,

Address correspondence and reprint requests to: Warren E. Lux, MD, U.S. Environmental Protection Agency, 1200 Pennsylvania Avenue, NW, Mail Code 8105R, Washington, DC 20460. E-mail: lux.warren@epa.gov. and coercion, for example, are among the actions considered ethically inappropriate in a way that transcends legal requirements, in that such actions fail in respecting the autonomy of the individuals who are their objects.

When the threshold for legal competency is not met and a surrogate must be appointed, actions not previously permitted on ethical grounds may be allowed by the surrogate- but only if ethically justified under the "substituted judgment," "best interests," or "reasonable treatment" standards. ${ }^{3}$ In the legal setting, moreover, the nature of these matters requires the use of a dichotomous approach to determining competency that incorporates a threshold determination. In the bioethical domain, a parallel and analogous approach to autonomy is critical to the ethical justification of actions that would not otherwise be morally acceptable.

However, few philosophers, if any, would seriously defend an account of autonomy that regarded autonomy itself as an inherently dichotomous property of either acts or persons. Rather, observations in both normal individuals and persons with neurological impairment suggest that the richness and complexity of human behaviors fall along a broad continuum of more or less autonomous actions. Moreover, ascriptions of autonomy to persons vary widely not only among individuals but also within the same individual as time, place, and situation change. 
A related observation from a more specifically clinical perspective is that autonomous actions require an array of cognitive capacities that are present to varying degrees in different individuals and that are to varying degrees activated and applied by the same individual at different times and in different circumstances.

Traumatic brain injury (TBI) can affect cognitive capacities critical to the effective exercise of autonomy. One of the notable features of this disorder is that TBI can produce survivors who retain normal or near-normal intellectual abilities, such that they may be adjudged to be competent and not in need of a guardian or other surrogate decisionmaker, but who nonetheless have an impaired ability to assess their own behaviors and capacities accurately. Accurate self-assessment, however, is critical to the maintenance and exercise of autonomy: it is a key faculty underlying self-correction and therefore genuine self-guidance. ${ }^{4}$ Moreover, the degree to which the actions of persons with impaired self-assessment actually stray off course as a consequence of that impairment may differ significantly from one environment to another, particularly in response to changes in environmental structure.

The picture is further complicated by the fact that when these individuals remain self-directed, they appear to be more self-guiding than they actually are. Furthermore, their impaired self-assessment causes them to think of themselves as more self-guiding than the facts justify. Thus, both the ascription of autonomy by others and the assertion of autonomy by the individual may be subject to error.

These changes can occur after TBI with a severity and persistence that warrants a finding of legal incompetence and the appointment of a guardian, but they can also occur at a level that does not warrant such an action. In the latter circumstance, the legal status of these individuals is decoupled from their autonomy status to a degree that varies with the fluctuations in their genuine selfguidance. As a consequence, the ethical framework that should guide the actions of family, friends, caregivers, and others in their interactions with these persons is not always straightforward and is worth examining more closely.

In order to raise some of the ethical issues and questions in a preliminary but grounded way, this article will first examine the neuropathology of TBI and the clinical neurocognitive changes that result as a basis for understanding how and under what circumstances autonomy may be compromised in these individuals. That review will be followed by the presentation of a hypothetical case derived from the composite clinical experience of the author that will highlight the practical difficulties, which will in turn lead to a presentation of some of the relevant ethical considerations.

\section{THE NEUROPATHOLOGY OF TBI AND ITS COGNITIVE CONSEQUENCES}

\section{Axonal strain injuries}

When the head is accelerated and decelerated in space, particularly when accompanied by a torsional component, strain forces are applied to axons throughout the brain. ${ }^{5,6}$ The resulting axonal strain injuries are proportional in amount to injury severity and represent the primary, fundamental neuropathological change seen in TBI due to closed mechanisms. ${ }^{7}$ Blunt-force trauma produces particularly violent acceleration-deceleration phenomena, so the most severe injuries tend to be produced by high-speed head impacts against immoveable objects as in falls from height and motor vehicle accidents. ${ }^{7}$

The anatomic and physiologic consequences of axonal strain are multiple. There may be axotomy, manifested by irreversible axonal shearing injuries, including gross injuries that are immediately visible on neuroimaging studies. Delayed axotomy secondary to evolving pathological processes set off by the initial injury has also been well demonstrated. ${ }^{8}$ Axons damaged at a level that does not cause frank axotomy may nonetheless show alterations in electrophysiological function. ${ }^{6}$ Other aspects of neuronal physiology may be altered as well, including acute and chronic levels of monoaminergic neurotransmitters. ${ }^{9-13}$ Moreover, these neurochemical changes can be correlated clinically with a number of well-documented post-TBI syndromes in which monoaminergic function has been implicated. ${ }^{14-17}$

The axonal injuries described here are called diffusewhich may be something of a misnomer, for although these injuries are multifocal and widespread, they are not uniformly distributed throughout the brain. The brainstem, particularly the upper brainstem, may be subjected to strains greater than would otherwise be expected, because of the relatively small attachment of the mesencephalon to the large masses of the cerebral hemispheres, and the specific biomechanics of an individual traumatic event will also modify the exact injury distribution. ${ }^{6}$ In addition, as injury severity increases and supratentorial axonal strain injuries accumulate, the injury shows an anterior-posterior gradient, at least in humans. Although the differences in gross brain anatomy make animal models less informative here, human clinical experience suggests that this gradient is an important phenomenon. Supporting evidence comes particularly from the neuroimaging literature, which has demonstrated that frontal and anterior temporal lesions are significantly more frequent than lesions more posterior in the cerebral hemispheres. ${ }^{18-20}$

\section{The neurocognitive consequences}

In the neurocognitive realm, the pathological changes described in the previous section translate first into impairment of what has been called power function (the 
concept is elaborated in the next section), due to the widespread axonal inefficiency resulting from the axonal strain injuries and their physiological consequences, and second into impairment of executive function and selfawareness, due to the anterior-posterior gradient. Note that the more posterior perceptual-motor and intellectual functions may be relatively well preserved, compared with these other functions, and may even be spared altogether.

Power function. The most fundamental neurocognitive disturbance seen after closed TBI is the impairment in power function, a term that refers to the basic mental functions necessary for the brain to engage in any cognitive task, regardless of what that task may be. The two critical components are arousal and channel capacity, and alteration in arousal at onset is a primary feature of closed TBI. Once arousal recovers, however, the clinical picture is dominated by the channel capacity disturbance. This disturbance is characterized clinically by difficulties in three important domains: processing speed, multitasking, and cognitive endurance. Virtually all persons who are symptomatic after TBI of any severity will demonstrate impairments in these domains, whether they have additional deficits or not.

When the deficit is severe enough to cause symptoms, the impairment in cognitive processing speed usually presents directly as slowed thinking. Even in asymptomatic patients, however, a slowing of cognitive processing speed can be demonstrated. ${ }^{21}$

The multitasking deficit commonly translates itself into difficulty performing a cognitive task efficiently in a distracting environment, where mental suppression of distraction and performance of the target task are required simultaneously. The patient's complaint may reflect this type of problem directly ("I can't think clearly when there's too much going on around me"), but it may relate instead to the cognitive domain to which the task itself belongs. The patient may say, for example, "I can't concentrate" or "I'm having problems with my memory," yet show good memory and attention if placed in a stimulus-controlled environment. It is simply that the patient cannot actually use the complex systems required for memory and attention effectively when subject to a multitasking requirement, even if there is no primary impairment in any of the specific components of the underlying systems.

That is not to say that TBI does not affect primary components of the attention and memory systems directly, particularly in the more severe injuries in which there is a greater burden of structural frontal and temporal damage. However, it is important to highlight how fundamental the disturbance in power function is to many of the cognitive complaints that arise after TBI. It may be the sole cause of cognitive symptoms. Even when it is not, and when there are primary impairments in other cognitive domains, the power function disturbance has the potential to exacerbate symptom severity, perhaps markedly.

The impairment in processing speed shares this property, as one would expect. So too does the impairment in cognitive endurance, although in that case the clinical presentation, like that of slowed processing speed, is usually straightforward. Symptomatic TBI patients are often simply unable to sustain any cognitive effort for as long as before the injury. Related to this reduction in cognitive endurance is the frequent complaint that life in general, and cognitive tasks in particular, are more effortful. Evidence in support of an organic basis for this complaint was provided in a recent functional magnetic resonance imaging study by McAllister et al. ${ }^{22}$ Performance on a cognitive task was equivalent for both the experimental (TBI) group and the control (normal) group, but the experimental group activated more cortex in doing so.

A self-evident final point about power function is that a breakdown in otherwise uncompromised cognitive domains in the face of inadequate power function is most likely to occur in those domains that are complex and particularly difficult or effortful to execute even under conditions of full power. Thus, if such a domain is also in fact compromised by the injury, the power function disturbance has the potential to have an even more devastating effect. In the types of TBI patients under discussion here, executive function is precisely such a domain.

Executive function. The term executive function refers to that set of neurocognitive capacities that are activated when one plans, initiates, and performs a goaldirected activity over time with appropriate self-monitoring and self-correction as one proceeds. Among the key components of executive function are anticipation, goal selection, planning, initiation, sequencing, monitoring (error detection), and self-correction (initiation of novel responses). ${ }^{23}$ It gives structure and coherence to human action in novel situations and is necessary for performing goal-directed activities successfully in unstructured real-world settings. Executive function relies on the activity of the frontal lobes anterior to the primary motor cortex, so it is vulnerable to the anterior-posterior gradient of structural brain lesions in TBI, particularly as injury severity increases.

Closely related to the primary components of executive function is the capacity for self-assessment. The importance of this capacity to executive function derives from the fact that effective task execution is possible only when one has the ability to accurately assess whether one has the physical and mental skills required to perform an intended act. Consider, for example, the case of a woman who wants to get from one place to another a few feet away but has severe leg weakness and 
is unable to ambulate without assistance. If she cannot accurately assess her ability to bear weight on her legs and as a result believes that she can walk independently when she cannot, she will fall when she tries to ambulate. If, on the other hand, accurate self-assessment is preserved, she will use crutches or a wheelchair or get help from an attendant and so succeed in executing her intended task.

Mental as well as physical capacities are subject to self-assessment, including the components of executive function itself. Thus, if someone is unable to detect errors well, for example, and is at the same time unable to assess one's own error-detection capacity, then multiple and even fatal errors are possible during task execution. If accurate self-assessment is preserved, however, and one knows that one has a problem with error detection, a number of strategies are available, such as double-checking one's work or seeking contemporaneous review by others.

The impaired self-awareness associated with selfassessment disorders in frontal TBI patients has some additional distinguishing features relevant to the capacity of these individuals to act autonomously. In particular, these individuals may in fact be able to recognize their deficits when the deficits are demonstrated to them, particularly in a highly structured setting. In that regard, their self-awareness deficits differ from those typically seen in patients with parietal (particularly right parietal) disease.

In the parietal cases, patients appear unable to perceive their deficits. In the frontal cases, on the other hand, ability to perceive the deficit is preserved, but there is a loss of the capacity to incorporate the knowledge of the perceived deficit into a global sense of one's self as an agent of action. Thus, persons with this syndrome canand do-acknowledge deficits that are demonstrated to them in concrete situations, but their subsequent behavior, verbal and otherwise, often does not reflect an awareness of the deficits in any meaningful way. As a consequence, they will say and do things in novel situations as if they did not in fact have the deficits that they were earlier able to acknowledge. ${ }^{4}$

To express it in a slightly different way, the primary disturbance in the TBI cases under consideration is in the ability to use available data about one's self to build an adequately accurate sense of who one is as a person. That is, the difficulty does not lie with the data themselves nor with the ability to acquire them in the first place. Thus, although autonomy may be compromised in both the parietal and the frontal cases, the mechanisms and clinical features are distinguishable, and the frontal patients are particularly alienated from themselves as agents of actions precisely when the opposite is required for genuine self-guidance.

\section{A HYPOTHETICAL CASE}

The patient under consideration in a matter of treatment refusal is a 24-year-old man who sustained a closed head injury in a motor vehicle accident 3 weeks earlier. He was unconscious at the scene, and his Glasgow Coma Scale score on admission to the emergency room was 9 . Computed tomographic scanning of the brain on admission showed a few bilateral frontal punctuate white matter hemorrhages, perhaps reflective of some gross axonal shearing lesions, but no surgical lesions. No MRI scan was done. Apart from the brain injury, there were no other significant injuries.

The patient emerged from coma on the third hospital day and went through an agitated phase lasting approximately a week. During this period, he was grossly confused and disoriented but was medically stable enough to be transferred to the hospital's rehabilitation unit. As his agitation subsided, his confusion also began to clear slowly but progressively. By the end of his third week in the hospital, the patient was alert and fully oriented. He was also freely ambulatory, albeit with a high-level balance disorder and problems with fine motor control. He was attending a full schedule of therapies.

Abnormalities on his cognitive examination included findings in the area of executive function. He had difficulty organizing and sequencing his day, but was able to compensate with the use of a day timer. This compensation would occasionally break down, however, resulting in missed therapy appointments, either at the end of the day when he was fatigued or when he had been off the rehabilitation unit and subject to the less structured and more distracting environment of the general hospital.

The patient's depth of awareness of his own capacities was shallow, and he had little insight into either his cognitive changes or his mild motor disorder, although he was able to acknowledge both when they were demonstrated to him. Memory and attention were impaired only when he was subjected to environmental distractions. Otherwise, his cognitive examination was remarkably intact. Language expression and comprehension were normal, as was calculation ability. Object recognition and constructional abilities were likewise normal. Geographic orientation was intact, and he was able to find his way around the rehabilitation unit and throughout the entire hospital without difficulty. He was able to understand facts and to reason logically from them. Moreover, he was fully self-directed, and although he was not cognitively normal, there was no question of his competence in a legal sense. Indeed, the question of seeking guardianship never arose after the acute phase of his injury was behind him.

His social history revealed that he was married and had an 18-month-old daughter. His wife, a beautician, had stopped working just prior to the birth of their child. 
At the time of his accident, the patient was employed as an auto mechanic at a local car dealership. The patient's parents lived nearby and were supportive. Prior to his accident, the patient had lived an essentially normal life. $\mathrm{He}$ did not smoke and used alcohol in moderation. He was calm in temperament, well-liked by his friends, and regarded as a good husband and father.

At this point, the patient decided that he wanted to stop all therapy and return home. He argued that his remaining deficits were minimal, and that it was his decision and intent to return to work "as soon as his employer would have him back." The treatment team recommended an additional one to two weeks of inpatient rehabilitation, to be followed by an outpatient work reentry program. The latter program would be specifically designed to deal with both the cognitive and the motor changes that he acknowledged and that affected his ability to do his work but that he was unable to incorporate into his greater sense of self as an agent. The program would also work with his employer to coordinate his return to work under structured, stimulus-controlled conditions that would maximize his chance of success. His wife and parents, more aware of the patient's changes than he was himself, also wanted to see him complete this course of treatment.

\section{SOME ETHICAL CONSIDERATIONS}

What actions are ethically justified on the part of the treatment team and the patient's family at this point? Should they accede to his decision, which he is legally entitled to make, allow him to leave the hospital, and try to develop an alternate treatment plan even though it might offer a lesser chance of success? Or should an attempt be made to keep him in the hospital against his expressed wishes and contrary to his own decision on the grounds that his decision is not fully autonomous because of his compromised executive function and selfawareness? And if the latter, what tactics are ethically permissible to accomplish this?

If a structured treatment contract presented in a stimulus-controlled setting in which deficits can be demonstrated compensates for his executive dysfunction such that he is able to make an arguably more autonomous decision to remain in the hospital, how far is his family permitted to go in encouraging him to accept the contract? In real situations of this sort, families commonly resort to coercive rhetorical pressure, sometimes with considerable force and persistence. Is this ever ethically justified, and if so, under what circumstances?

Raising these issues as they emerge in this kind of case, particularly against the background of the neurocognitive analysis of TBI, highlights two ethically relevant points, as introduced at the outset of this review. The first is the distinction between autonomous acts and autonomous persons, a distinction that has always been apparent but that takes on notable ethical relevance in the context of this kind of decision-making.

Individuals with TBI who possess many or most of the capacities of autonomous persons, such that they are justifiably adjudged to be legally competent, may nonetheless have deficits that lead them to engage in individual actions and make particular choices in ways that are clearly not autonomous. Moreover, it can be argued that normal individuals without such deficits also do the same thing at times. In such cases, however, it is usually a more inconsistent occurrence, whereas individuals with TBI have a propensity for this to happen on the basis of particular deficits. In either case, however, application of the principle of respect for autonomy requires an analysis of the act in question and cannot be adequately addressed by exclusive reference to the overall autonomy of the person. Beauchamp and Childress ${ }^{2}$ make this same point. The current example of TBI serves to highlight it.

The second point is that autonomy failure is sensitive to the situational elements that surround it. Again, this is true of both normal individuals and persons with TBI. Using the TBI example, however, allows many of these elements to be defined clinically and further understood on the basis of neurocognitive analysis. Although this may not inform all instances of autonomy failure in normal persons-or even in persons with TBI, for that matter-it may be helpful in at least some cases. In addition, it implies that autonomy failure is treatable, in that it can respond to appropriate environmental interventions directed at those situational elements that are undermining autonomy.

Thus, the simple expedient of changing the decisionmaking milieu from a distracting environment to a quiet, stimulus-controlled environment may suffice to convert a nonautonomous decision into an autonomous one. Moreover, an ethical imperative to apply interventions that can have such results is implied by the principle of respect for autonomy for both normal persons and those with cognitive impairments.

Given the particular impairments in this case, and the ethical considerations that derive from the case, successful application of treatment interventions may involve the participants in a striking paradox. TBI and conditions like it may produce circumstances that would lead one to consider tactics (such as coercion, in this example) that compromise the autonomy of the person in the short term in order to enable autonomous choice later when it would otherwise be precluded.

Justifying such a tactic would have to include, at a minimum, the aim of reestablishing the relative autonomy of the person over the longer term and to a degree that would not otherwise occur. There should be reasonable evidence for the clinical efficacy of the proposed intervention in the particular case under consideration, 
and that evidence would need to be brought to bear with an Aristotelian sort of prudential judgment in the ethical as well as the clinical realm.

Acknowledgments: Some of the ideas contained in this work were first presented at a problem case conference at the Center for Clinical Bioethics at Georgetown University in September 2002 and later at a symposium on the neuroethics of brain damage held at Washington University in St. Louis in December 2002. I have benefited from discussions with colleagues at both institutions, and their critical feedback is gratefully acknowledged. I would also like to acknowledge my colleagues in the Office of the Science Advisor at the U.S. Environmental Protection Agency for their support of a work culture in which ideas matter. The views expressed in this article, however, are solely those of the author and do not reflect official positions of the U.S. Environmental Protection Agency nor of the U.S. Government.

\section{REFERENCES}

1. Frankfurt HG. Autonomy, necessity, and love. In: Necessity, volition, and love. Cambridge: Cambridge University Press; 1999: 129-141.

2. Beauchamp TL, Childress JF. Respect for autonomy. In: Principles of biomedical ethics, 5th ed. New York: Oxford University Press; 2001:57-112.

3. Devettere RJ. Standards for making proxy decisions. In: Devettere RJ. Practical decision making in health care ethics: cases and concepts, 2nd ed. Washington, DC: Georgetown University Press; 2000:132-138.

4. Anderson J, Lux W. Knowing your own strength: accurate selfassessment as a requirement for personal autonomy. Philos Psychiatry Psychol 2004;11:279-294.

5. Ommaya AK, Gennarelli TA. Cerebral concussion and traumatic unconsciousness: correlation of experimental and clinical observations of blunt head injuries. Brain 1974;97:633-654.

6. Gennarelli TA. Mechanisms and pathophysiology of cerebral concussion. J Head Trauma Rehabil 1986;1:23-29.

7. Alexander MP. Mild traumatic brain injury: pathophysiology, natural history, and clinical management. Neurology 1995;45:12531260.

8. Povlishock JT. Pathobiology of traumatically induced axonal injury in animals and man. Ann Emerg Med 1993;22:980-986.

9. Bareggi SR, Porta M, Selenati A, et al. Homovanillic acid and 5-hydroxyindole-acetic acid in the CSF of patients after a severe head injury: I. Lumbar CSF concentration in chronic brain posttraumatic syndromes. Eur Neurol 1975;13:528-544.
10. Porta M, Bareggi SR, Collice M, et al. Homovanillic acid and 5-hydroxyindole-acetic acid in the CSF of patients after a severe head injury: II. Ventricular CSF concentrations in acute brain post-traumatic syndromes. Eur Neurol 1975;13:545-554.

11. Vecht CJ, van Woerkom TCAM, Teelken AW, Minderhoud JM. 5-Hydroxyindoleacetic acid (5-HIAA) levels in the cerebrospinal fluid in consciousness and unconsciousness after head injury. Life Sci $1975 ; 16: 1179-1185$.

12. Vecht CJ, van Woerkom TCAM, Teelken AW, Minderhoud JM. Homovanillic acid and 5-hydroxyindoleacetic acid cerebrospinal fluid levels: a study with and without probenecid administration of their relationship to the state of consciousness after head injury. Arch Neurol 1975;32:792-797.

13. van Woerkom TCAM, Teelken AW, Minderhoud JM. Difference in neurotransmitter metabolism in frontotemporal-lobe contusion and diffuse cerebral contusion. Lancet 1977;1:812-813.

14. Jorge R, Robinson RG. Mood disorders following traumatic brain injury. Int Rev Psychiatry 2003;15:317-327.

15. Fedoroff JP, Starkstein SE, Forrester AW, et al. Depression in patients with acute traumatic brain injury. Am J Psychiatry 1992; 149:918-923.

16. Berthier ML, Kulisevsky J, Gironell A, Heras JA. Obsessivecompulsive disorder associated with brain lesions: clinical phenomenology, cognitive function, and anatomic correlates. Neurology 1996;47:353-361 [Erratum in: Neurology 1996;47:855].

17. Hollander E, Wong CM. The relationship between executive function impairment and serotonergic sensitivity in obsessive-compulsive disorder. Neuropsychiatry Neuropsychol Behav Neurol 1996; 9:230-233

18. Levin HS, Williams D, Crofford MJ, et al. Relationship of depth of brain lesions to consciousness and outcome after closed head injury. J Neurosurg 1988;69:861-866.

19. Levin HS, Mendelsohn D, Lilly MA, et al. Magnetic resonance imaging in relation to functional outcome of pediatric closed head injury: a test of the Ommaya-Gennarelli model. Neurosurgery 1997;40:432-441.

20. van der Naalt J, Hew JM, van Zomeren AH, Sluiter WJ, Minderhoud JM. Computed tomography and magnetic resonance imaging in mild to moderate head injury: early and late imaging related to outcome. Ann Neurol 1999;46:70-78.

21. Warden DL, Bleiberg J, Cameron KL, et al. Persistent prolongation of simple reaction time in sports concussion. Neurology 2001;57: 524-526.

22. McAllister TW, Saykin AJ, Flashman LA, et al. Brain activation during working memory 1 month after mild traumatic brain injury: a functional MRI study. Neurology 1999;53:1300-1309.

23. Lezak MD. Assessment of psychosocial dysfunctions resulting from head trauma. In: Lezak MD, editor. Assessment of the behavioral consequences of head trauma. New York: Alan R. Liss; 1989:113-143. 